\title{
Drug Shortages: The Survey Says ...
}

\author{
Douglas Sellinger
}

$\mathrm{M}$ y daughter is graduating from high school and has completed her university application. She will be entering a pre-pharmacy program and is thinking about a career in hospital pharmacy, so perhaps the apple really doesn't fall too far from the tree. I recall the upheaval that her starting school a dozen years ago created in our family. Every year, she became involved in more scholastic and extracurricular activities. As the associated parental work increased, our ability to accomplish the tasks also grew, in part because we gave up other activities. In many ways, the Canadian drug shortage situation has had the same effect on pharmacists.

The Canadian Society of Hospital Pharmacists (CSHP) recently joined the Canadian Pharmacists Association and the Canadian Medical Association in surveying members about drug shortages and releasing a statement about the results to the media. ${ }^{1}$ If you missed it on your local news broadcast, don't feel bad: media pick-up of the story was limited and so was the interest of our politicians. Perhaps what was missing from the story was the aspect of potential patient harm. The diligent and timely mitigation of drug shortages by hospital and community pharmacists and by physicians has resulted in many positive patient outcomes, but these tend not to make great news stories.

Survey results indicated that up to $20 \%$ of patients have been affected by shortages. The top 3 impacts for patients were delayed access to needed medication, use of a less effective medication or formulation, and increased risk of adverse effects. Hospital pharmacists who responded to the survey indicated that patients experienced delays in acquiring their medications, additional cost associated with alternative medications, and confusion related to changes in medication or formulation.

Of 215 hospital pharmacists who responded, more than half reported trouble sourcing a medication for a patient during their most recent shift, and almost two-thirds indicated difficulty getting a medication in the previous week. Hospital pharmacists' strategies to mitigate the effects of drug shortages included communication within the pharmacy, hospital, and health region; rationing or restricting the use of existing supplies; obtaining medications from another source; compounding from an alternative concentration or formulation of the same medication; changing the route of administration; or altering therapy to a medication not currently in shortage. All of these strategies take time, often the pharmacist's time and often at the expense of other activities, including the delivery of direct care to patients.

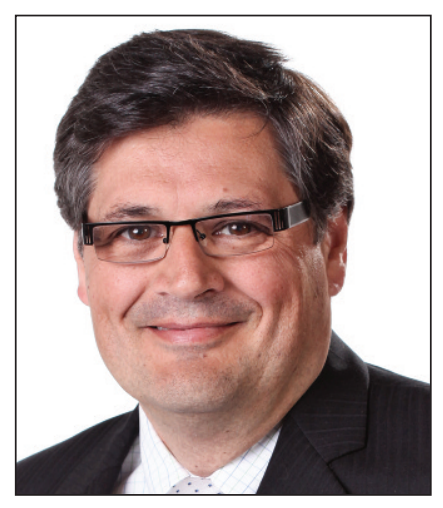

There has been a paradigm shift in health care, with drug shortages becoming part of everyday life, and hospital pharmacists are on the front line of this shift. The opportunity exists to create effective, clinically appropriate medication-use strategies for both shortage and normal-supply situations. Patients rely on our expertise in medication management and our compassion to aid them on their journey through the dark forest of drug shortages.

Although CSHP appreciates that drug shortages require a global solution, the Society is working here in Canada with multiple stakeholders, including federal, provincial, and territorial governments, to reduce the occurrence of drug shortages and to mitigate their effects on patients and health care professionals. Through these efforts, I hope that CSHP will be able to reduce the impact, stress, and anxiety of drug shortages on hospital pharmacists and their patients.

\section{Reference}

1. Canadians paying the price for drug shortages: survey [media release]. Ottawa (ON): Canadian Pharmacists Association; 2013 Jan 14 [cited 2013 Feb 25]. Available from: www.pharmacists.ca/index.cfm/news-events/news/ canadians-paying-the-price-for-drug-shortages-survey/

Douglas Sellinger, BSP, MALT, is the President and External Liaison for the Canadian Society of Hospital Pharmacists. 